\title{
CAUSAS O AZARES: REFLEXIONESENTORNOA LAPRESENCIA DE MALFORMACIONES CONGÉNITAS DE LA COLUMNA VERTEBRAL EN POBLACIONES ARQUEOLÓGICAS DE LA COSTA DESÉRTICA DE ANTOFAGASTA, NORTE DE CHILE
}

\author{
CAUSALITY OR RAMDOMNESS: CONSIDERATIONS ON THE PRESENCE \\ OF CONGENITAL MALFORMATIONS ON THE SPINAL COLUMN IN \\ ARCHAEOLOGICAL POPULATIONS OF THE DESERTIC COAST OF \\ ANTOFAGASTA, NORTHERN CHILE
}

\author{
Pedro Andrade ${ }^{1,2 *}$ y Monserrat Araneda ${ }^{3}$ \\ ${ }^{1}$ Carrera de Antropología, Universidad de Concepción. Concepción, Chile \\ ${ }^{2}$ Programa de doctorado UTA-UCN \\ ${ }^{3}$ Carrera de Antropología, Universidad de Concepción. Concepción, Chile
}

PALABRAS CLAVES: costa desértica de Antofagasta; enfermedad congénita; sacralización lumbar; cazadores-recolectores; endogamia

RESUMEN La sacralización de la quinta vértebra lumbar corresponde a una patología escasamente reportada en la literatura bioarqueológica de las poblaciones del Norte Grande de Chile. En este artículo, se presentan dos casos observados en grupos prehispánicos de la costa desértica de la región de Antofagasta, distanciados tanto espacial como cronológicamente; uno de ellos corresponde al caso más temprano de esta anomalía ósea para el norte de Chile. El diagnóstico de esta condición fue realizada a través de criterios discriminantes morfológicos que permitieron la clasificación de forma y orientación de los rasgos patológicos observados. Considerando la asociación a factores genéticos que posee la manifestación de esta patología, junto con la descripción histórica de las formas de elaboración de alianzas matrimoniales que tenían los habitantes del área de estudio al momento de contacto hispano-indígena, en este trabajo exploramos la posibilidad de que su aparición pueda ser el resultado de prácticas sociales endogámicas. Estas habrían estado limitadas a la zona costera y fueron mantenidas tradicionalmente, por lo menos hasta momentos históricos. Rev Arg Antrop Biol 22(2), 2020. doi: 10.24215/18536387e019

KEYWORDS desertic coast of Antofagasta; congenital disease; lumbar sacralization, hunter-gatherers; endogamy

ABSTRACT The sacralization of the fifth lumbar vertebra corresponds to a pathology scarcely reported in the bioarchaeological literature for the northern populations of Chile. In this paper, two cases observed in pre-Hispanic groups of the desert coast of the Antofagasta region are presented, which are both spatially and chronologically distant from each other, and one of them corresponds to the earliest case of this anomaly reported for northern Chile. The diagnosis of this condition was made through discriminating morphological criteria that allowed the classification of form and orientation of the pathological features observed. Considering the association between the manifestation of this pathology and genetic factors, together with the historical description of the marriage alliances forged by the inhabitants of the study area at the time of indigenous-Hispanic contact, we explored the possibility that its manifestation could be the result of endogamic social practices. The latter might have been limited to the coastal area and were traditionally maintained at least until historical times. Rev Arg Antrop Biol 22(2), 2020. doi: 10.24215/18536387e019
La costa de la región de Antofagasta (21 ${ }^{\circ} 26^{\prime}$ a $26^{\circ} 03^{\prime}$ 'Lat. Sur) corresponde a una zona desértica agreste, caracterizada por una falta de cursos fluviales permanentes que desemboquen en el mar, un estrecho espacio litoral y baja productividad del suelo (Pliscoff y Luehbert, 2008; Quezada, Cerda y Cerda, 2010). Se suman a estas condiciones la ocurrencia de eventos catastróficos naturales, como terremotos y el fenómeno de El Niño (Vargas, Rutland y Ortlieb, 2006; Vargas et al., 2011). A pesar de estas circunstancias, esta zona posee una riqueza y alta productividad de recursos marinos

Financiamiento: Proyecto FONDECYT 1151203 - Proyecto FONDECYT 1100951

*Correspondencia a: Pedro Andrade. Universidad de Concepción. Víctor Lamas 1290, Concepción, Chile.

E-mail: pandradem@udec.cl

Recibido 30 Mayo 2018; aceptado 14 Septiembre 2019

doi:10.24215/18536387e019 
(Ortlieb, Vargas y Saliege, 2011), las cuales han sido la base de la subsistencia de las poblaciones humanas que han habitado este espacio de manera casi permanente desde hace 12.000 años (Andrade et al., 2015; Pestle, 2017; Pestle, Torres-Rouff, Ballester, Gallardo y Clarot, 2015). Dichas poblaciones han sido caracterizadas como cazadores-recolectores-pescadores durante gran parte de la secuencia ocupacional prehispánica (Castro, 2014; Salazar et al., 2015). Producto de esta extensa cronología de ocupaciones humanas, existe un amplio registro de sitios arqueológicos a lo largo de la costa de Antofagasta, donde se incluyen sectores de entierros humanos aislados, hasta sectores de cementerios (Ballester y Clarot, 2014; Castelleti y Maltrain, 2010; Costa-Junqueria, 2001; Núñez, Zlatar y Núñez, 1975). Lamentablemente a lo largo de la costa de Antofagasta, muchos de estos contextos funerarios se encuentran amplia y profusamente saqueados (Andrade, Salazar, Urrea y Castro, 2014; Llagostera y Cruz, 2010; Torres Rouff, 2017). A pesar de esto, los trabajos sistemáticos de distintos grupos de investigación en la zona (Andrade, Castro y Aldunate, 2016; Andrade et al., 2014; Ardiles, Ballester y Clarot, 2011; Arias y Herrera, 2012; Ballester, Clarot y Llagostera, 2014; Ballester et al., 2018; Berrios, 2014; Clarot, Méndez y Ballester, 2014-2015; Cocilovo, Varela, Costa-Junqueira y Quevedo, 2005; Constanzo, 2013; Costa-Junqueira y Sanhueza, 1976; Munizaga, 1980; Torres-Rouff, 2017) han permitido conocer distintos aspectos de los modos de vida de los antiguos habitantes del desierto costero de Antofagasta.

En el presente trabajo presentamos el hallazgo de dos individuos que han sido recuperados en distintos sitios y contextos cronológicos pertenecientes a la zona costera antofagastina, y presentan evidencias de sacralización incompleta de la quinta vértebra lumbar. Esta corresponde a un tipo de vértebra en transición lumbosacra (o LSTV por sus siglas en inglés, abreviación que será utilizada a lo largo del texto), clasificada dentro de las anomalías congénitas que afectan a la columna vertebral. Esta patología a nivel mundial se encuentra ampliamente documentada, siendo su caso más temprano proveniente de un individuo Neanderthal (Aufderheide y Rodriguez-Martin, 1988). Si bien en la literatura especializada, la mayo- ría de los casos se reportan en el Viejo Mundo (Aufderheide y Rodriguez-Martin, 1998; Brothwell, 1987; Roberts y Manchester, 2005, entre otros), también existen casos de estudio en el territorio americano (Barnes, 2008; Caldwallader y Arce, 2012; Kozameh, Testa y Tessone, 2011; Miranda, 2011; Salgan, Tucker, Luna, Aranda y Gil, 2012). En la costa norte de Chile, solo existe un caso reportado para el periodo Intermedio Tardío de Arica (1000-1400 d.C.) (Arriaza, Standen, Soto y Aravena, 2019), mientras que, para la costa de Antofagasta, su presencia aun no ha sido reportada en la literatura arqueológica, por lo que se considera relevante reflexionar en torno a las causas de su aparición. Entre las posibles explicaciones en torno a las causas de expresión de las LSTV, las mismas pueden ser el resultado de: 1) factores ambientales ligados al consumo de metales tóxicos presentes en las escasas fuentes de agua disponibles en la zona, y/o 2) un sistema de parentesco endogámico de larga data. Dadas las descripciones etnohistóricas e históricas relativas a la endogamia que presentaban las poblaciones costeras de la región de Antofagasta, en este trabajo se evaluará la posibilidad de que la expresión de las LSTV en los grupos humanos prehistóricos de la zona de estudio sea causa de factores relacionadas con un sistema de parentesco endogámico de larga data.

\section{Cronología y contextualización general de la prehistoria de la costa de Antofagasta}

Dentro de las cronologías propuestas para la zona de estudio (Castelleti, 2007; Castro, 2014; Llagostera 2005; Salazar et al., 2015) se reconoce una larga secuencia ocupacional que se inicia hace unos 11.500 años atrás con el periodo Arcaico (Salazar et al., 2018), el cual se prolongará por casi 9.000 años. Esta extensa etapa está marcada por la presencia de grupos cazadores-recolectores litorales especializados en la extracción de recursos marinos y en la minería, desarrollando complejos conjuntos artefactuales en piedra y hueso, además de la navegación (Flores, Figueroa y Salazar, 2016; Olguín, Salazar y Jackson, 2014; Salazar et al., 2011). Se ha planteado previamente que estos grupos humanos habrían tenido un origen común con aquellas 
poblaciones de la costa de Arica, aunque debido a las condiciones geográficas de aislamiento, habrían evolucionado diferenciándose genéticamente de sus vecinos costeros al norte y al sur (Cocilovo, Varela, Quevedo, Standen y Costa-Junqueira, 2004, Cocilovo et al., 2005; Henríquez, Moraga, Llop y Rothhammmer, 2004; Ibacache, 2014). Unos 2.000 años atrás comienza el periodo Formativo (Gallardo, 2017), el cual se caracteriza por la intensificación de contactos e intercambio de productos con grupos de San Pedro de Atacama y de Tarapacá. Esto resulta en la llegada de artefactos novedosos hacia la costa, tales como la metalurgia, cerámica, además de textilería y el complejo fumatorio (Carrasco, Carmona, Ballester y Niemeyer, 2013; Correa, Carrasco, Ballester y Gallardo, 2018; Pimentel, Rees, de Souza y Ayala, 2010). Este momento se caracteriza también por la presencia de monumentos funerarios, conocidos como túmulos, los cuales se encuentran en distintos sectores de la extensa costa desértica (Ballester y Clarot, 2014; Gallardo, Ballester y Fuenzalida, 2017). Hacia el año 1.000 d.C., se intensifica aún mas la presencia de materiales foráneos al área de estudio, apareciendo ya no solo materiales del interior de la región de Antofagasta, sino que también de sectores tan distantes como los Valles Occidentales de Arica y Parinacota (Castro, 2014). De esta manera, el sector desértico costero se configura como un espacio donde se habrían llevado a cabo relaciones sociales entre grupos de distintas etnias. Destacan de este momento las primeras manifestaciones de una producción alfarera local (Varela, 2008), como también la aparición de manifestaciones de arte rupestre conocido como estilo El Médano (Ballester, 2018; Berenguer, 2009).

El último momento de esta secuencia prehistórica corresponde al Periodo Tardío, marcado por la presencia de artefactos Incaicos, especialmente en los sectores del norte de la costa de Antofagasta (Castro, 2014; Salazar et al., 2010). No obstante, la presencia Incaica en la zona no habría sido permanente (Castro, Aldunate y Varela, 2012), sino que solo se habría establecido con el fin de generar redes de intercambio de recursos marinos y minerales con los grupos locales (Aldunate, Castro y Varela, 2010; Blanco, Correa, Flores y Pimentel, 2017). Cabe mencio- nar que, a lo largo de esta secuencia cronológica, los grupos humanos de la costa desértica de Antofagasta no habrían modificado su subsistencia sustancialmente (Andrade et al., 2015; Castro, 2014), lo cual se puede explicar por las particulares condiciones ambientales áridas de la costa, que no habrían permitido el desarrollo de la agricultura (Castro, 2014).

\section{Las prácticas endogámicas en las poblaciones históricas de la costa Arreica de Antofagasta}

Los estudios previos sobre las relaciones de parentesco de las poblaciones del norte de Chile, se han realizado principalmente a partir de datos recuperados del texto eclesiástico del siglo XVII denominado "Libro de Varias Ojas" de la parroquia de Chiu Chiu (Aldunate et al., 2010; Ballester, 2017; Ballester y Gallardo, 2017; Letelier y Castro, 2017), donde se han ido registrando las visitas, bautizos y matrimonios realizados en la zona durante momentos coloniales. En términos generales, dichos estudios han reconocido la nula existencia de matrimonios entre individuos costeros y del interior. Es decir, las estrategias reproductivas de los individuos de la costa habrían sido acotadas a la zona costera, donde los intercambios se habrían generado entre los distintos enclaves litorales. De esta forma, los habitantes de este sector, se reconocen como parte de un mismo sistema social, sin integrar a personas foráneas. En consecuencia, el esquema de parentesco muestra que las uniones matrimoniales se realizaban entre territorios reproductivos (sensu Ballester, 2017), donde se practicaba una exogamia a nivel residencial local, pero una endogamia a nivel macroespacial, la cual se manifestaba a lo largo de $500 \mathrm{~km}$ de línea costera (Ballester y Gallardo, 2017). Letelier y Castro (2017) plantean que esto se habría mantenido hasta tiempos republicanos, toda vez que la condición de endogamia regional costera se puede ver reflejada también en los datos obtenidos por los censos locales realizados en el siglo XIX. Estudios recientes sobre parentesco en comunidades modernas locales de la zona sur de la costa desértica de Antofagasta, reafirman lo planteado anteriormente (Castelleti, 2019; Castelleti, Villarroel, Almendares y Mercado, 2019). 


\section{Clínica y sintomatología de las LSTV}

Los defectos congénitos son anomalías estructurales o funcionales producidas durante el desarrollo intrauterino (Rojas y Walker, 2012). Para este estudio en específico se utilizará el concepto de Defecto del Desarrollo y no el de Trastorno Congénito (sensu Barnes, 1994), ya que el segundo término alude a la detección de la anomalía en el recién nacido, lo cual es un hecho poco común. En cambio, los defectos del desarrollo no se detectan sino cuando la condición se agrava durante el crecimiento y desarrollo, durante la infancia y/o adolescencia, o cuando existen causas relacionadas a estrés funcional o traumas durante la adultez (Goodman y Gorlin 1983).

Dentro de los defectos del desarrollo que se producen en la columna vertebral se encuentra el caso de las vértebras en transición lumbosacra o LSTV, que corresponden a defectos en la segmentación de la columna lumbosacra durante el desarrollo intrauterino (Murlimanju, Prabhu, Pai, Ganeshkumar y Sarvepalli, 2011). Castellvi, Goldstein y Chan (1984), clasificaron las LSTV en 4 tipos (Fig. 1): el tipo I es un proceso trans-

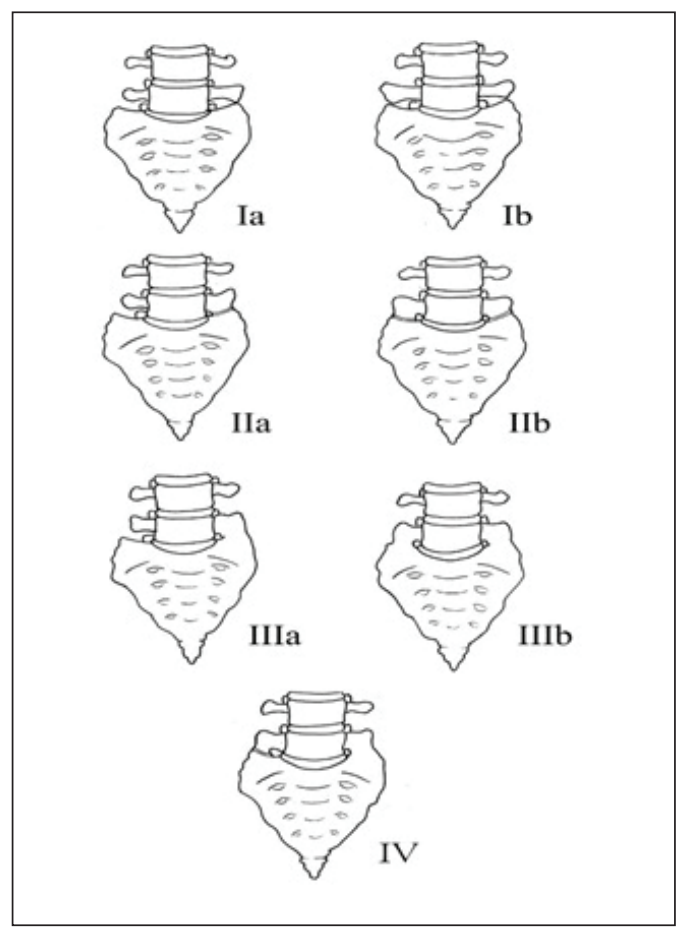

Fig. 1. Descripción gráfica de los cuatro tipos propuestos por Castellvi et al. (1984) verso displásico de forma triangular, menor de 19 $\mathrm{mm}$ de ancho, unilateral (tipo Ia) o bilateral (tipo Ib); el tipo II corresponde a una lumbarización o sacralización incompleta con pseudoartrosis en el ala sacra adyacente, unilateral (tipo IIa) o bilateral (tipo IIb); el tipo III presenta lumbarización o sacralización con el proceso transverso de tamaño aumentado y fusionado, unilateral (tipo IIIa) o bilateral (tipo IIIb); y el tipo IV (o mixto) corresponde a la presencia del tipo IIa en un lado del sacro y del tipo IIIa en el lado contrario.

A partir de esta clasificación se pueden diferenciar dos manifestaciones que, si bien pueden compartir características similares, también presentan diferencias: lumbarización de la primera vertebra sacra (lumbarización de S1), y sacralización de la quinta vértebra lumbar (sacralizacion de L5). La lumbarización de S1, definida como la presencia de cinco vértebras lumbares distintas con el proceso transversal de la primera vértebra sacra fusionándose o formando una pseudoartrosis con el ala sacra unilateral o bilateral. En la misma, S1 muestra signos de transición a una configuración lumbar, por lo que no es englobada en la osificación de las vértebras sacras, de manera que aparecen seis vértebras lumbares separadas y cuatro fusionadas en el sacro (Kumari, Kumari, Panchal, Prassad y Britto, 2016; Murlimanju et al., 2011; Sekharappa, Amritanand, Krishanan y Samuel, 2014). La sacralización de L5, constituye la fusión o semi-fusión del proceso transverso de L5 con el sacro, el ilion, o ambos, formando un puente óseo o pseudoartrosis con el ala sacra unilateral o bilateral. En este caso, L5 muestra signo de asimiliación y - en algunos casos - de fusión al sacro, de manera que aparecen cuatro vértebras lumbares y seis vértebras sacras (Kumari et al., 2016; Murlimanju et al., 2011; Sekharappa et al., 2014).

Con respecto al origen anatómico de las LSTV, este correspondería a un defecto en la expresión normal de los caracteres de la columna vertebral. En el caso del desarrollo embrionario de los seres humanos existe un grupo de genes conservados evolutivamente que codifican los factores que regulan los procesos morfogenéticos, denominados genes Hox, los cuales continúan su expresión incluso en la edad adulta (Quinonez e Innis, 2014). Los genes responsables del desarrollo de los elementos vertebrales 
son los genes parálogos Pax-1 y Pax-9, que interaccionan conjuntamente regulando el desarrollo del cuerpo vertebral, arco vertebral y disco intervertebral (LeClair, Bonfiglio y Tuan, 1999). Además, el gen parálogo Pax-11 regula el patrón morfológico del sacro, en conjunto con otros genes, particularmente el gen Pax-10 que controla el desarrollo de la columna vertebral (Carapuco, Nóvoa, Bobola, y Mallo et al., 2005). Experimentos genéticos en ratones han mostrado que las mutaciones que inhiben la función de los genes Hox, alteran a los tejidos localizados durante la expresión del gen mutado (Mallo, Wellik y Deschamps, 2010). Por lo tanto, la alteración en alguno de estos genes podría ser la causa de las malformaciones en la zona lumbosacra y, en consecuencia, originar las LSTV. Las mismas tienen como característica presentar aspectos altamente variables de individuo a individuo, lo que ha generado discusiones en términos de prevalencia poblacional, su asociación a otras patologías vertebrales, y su sintomatología (Barnes, 2008).

Respecto a su prevalencia se han reportado amplios rangos, los cuales oscilan entre el $4 \%$ y el $35 \%$ según la población estudiada (Hsieh, Vanderford, Moreau y Prong, 1999; Leboeuf, Kimber y White, 1989; Otani, Konno y Kikuchi, 2001; Sekharappa et al., 2014; Vergauwen et al., 1997; entre otros). No obstante, estudios extensos y sistemáticos basados en revisiones bibliográficas clínicas actuales, estiman una prevalencia de $12,3 \%$ para las LSTV, planteándose que al menos un $50 \%$ de los casos informados presentan una división clara entre la lumbarización y la sacralización, las cuales dentro de este total corresponderían al 5,5\% y al 7,5\% de los casos, respectivamente (Bron, 2007).

En cuanto a las patologías asociadas, se plantea que las LSTV son un factor de riesgo que pueden desencadenar cambios degenerativos en los cuerpos y discos vertebrales (Hsieh et al., 1999, Leboeuf et al., 1989), causando una reducción de la altura del disco intervertebral (Hsieh et al., 1999) o generando cargas de manera unilateral o asimétrica, provocando escoliosis por la inclinación y curvatura de la columna lumbar (Barnes, 1994). Por otro lado, en relación con enfermedades degenerativas articulares y las LSTV, Castellvi y colaboradores
(1984) estudiaron la asociación de LSTV y herniación, encontrando una alta incidencia de la primera en pacientes con herniación del disco $(83,4 \%)$. Resultados similares han sido descritos por Taskaynatan, Izci, Ozgul, Hazneci, Dursun y Kaylon (2005) y Otani et al. (2001) en pacientes con herniación del núcleo pulposo $(75 \%)$. Finalmente, se ha asociado la presencia de LSTV a la formación de espondilosis lumbosacra (de Bruin et al., 2017; Kong, Park y Park, 2008; Shekhrappa et al., 2014). Sin embargo, estudios realizados por Vergauwen y colaboradores (1997), discrepan en el hecho de que la relación entre las LSTV y los cambios degenerativos pueda ser significativa. Con respecto a la coexistencia de LSTV con otras alteraciones congénitas, Paraskeas, Tzikas y Kitsoulis (2013) estudiaron la relación entre LSTV y la espina bífida oculta del sacro en una población griega y encontraron coexistencia entre ambas patologías, aunque en porcentajes menores al $1 \%$.

Finalmente, el dolor generado en la zona lumbar que puede ser asociado a LSTV se conoce como Síndrome de Bertolotti (Moreno, del Río, Baltanás y Cía, 2016), y se asocia a los tipos II y IV, generando además dolor en el sector glúteo (Nardo et al., 2012; Tang et al., 2014). Específicamente para pacientes con lumbarización de $\mathrm{S} 1$, se indica la presencia de una alteración de la raíz nerviosa lumbosacra, lo cual genera dolor (Chang y Nakagawa, 2004). Sin embargo, Barnes (1994) plantea que cuando la lumbarización es bilateral y simétrica, no presentaría sintomatología dolorosa.

\section{MATERIAL Y METODOS}

En este estudio se presentan dos individuos recuperados de los sitios Hornos de Cal y Caleta El Fierro Alero 1 (CEFA-1) (Fig. 2). En el caso del primero, se trata de un contexto primario e individual en una capa estéril bajo un conchero de uso habitacional, correspondiente a una paleoplaya de conchilla. El individuo se encontraba dispuesto decúbito dorsal, con los miembros superiores e inferiores flectados, sin registrar ofrendas ni estructura funeraria asociada. Las unidades óseas presentaban un regular estado de conservación. Fechados obtenidos de este sitio, indican que el mismo ha sido ocupado entre los años 6.500 y $3.500 \mathrm{cal}$ AP (FONDECYT 
1151203, 2017). Por otro lado, el individuo de CEFA-1, se encontraba dispuesto dentro de un pequeño alero rocoso, que había sido saqueado, por lo que no fue posible distinguir su patrón funerario, aunque parece haber correspondido a un entierro individual. A pesar de todo lo anterior, las unidades óseas presentes se encontraban en buen estado de conservación. Si bien no se pudo determinar la presencia directa de ofrendas o de ajuar, se pudo encontrar restos de textilería junto a las unidades óseas. No se registró la presencia de más entierros directamente en este contexto funerario, aunque sí existen varios cementerios de túmulos en las cercanías.

La determinación del sexo y la estimación de edad de los especímenes se realizaron siguiendo las recomendaciones de Buikstra y Ubelaker (1994), considerando rasgos diagnósticos en el cráneo y los huesos coxales, para la primera, y el desarrollo de huesos largos, costillas, cráneo y erupción dental para la segunda. Para las patologías vertebrales se utilizó una metodología macroscópica, siguiendo las indicaciones de Campo (2003). Esta metodología permite registrar las anomalías que afectan distintos segmentos de la morfología normal de las unidades vertebrales, lo que ayuda a realizar un diagnóstico más acabado para las enfermedades congénitas, degenerativas, metabólicas, infecciosas y traumáticas que afectan a los diferentes segmentos de la columna vertebral. Para diferenciar los procesos de sacralización y lumbarización, se ocuparon los criterios de diagnóstico propuestos por Barnes (2008), que se basan en las distintas secciones morfológicas de L5 y de S1 para realizar la determinación de cada una de las patologías. Finalmente, se utilizó la clasificación de Castellvi y colaboradores (1984) para describir la forma y orientación de los procesos transversales, y los diversos grados de articulación entre el ala sacra y L5.

Complementariamente, se obtuvieron fechados radiocarbónicos directos de los individuos sobre fragmentos de fémur, los que fueron enviados al Center for Applied Isotope Studies, de la Universidad de Georgia, Estados Unidos, permitiendo situarlos cronológicamente dentro de la secuencia ocupacional regional. Estas fechas fueron calibradas con dos sigmas, mediante

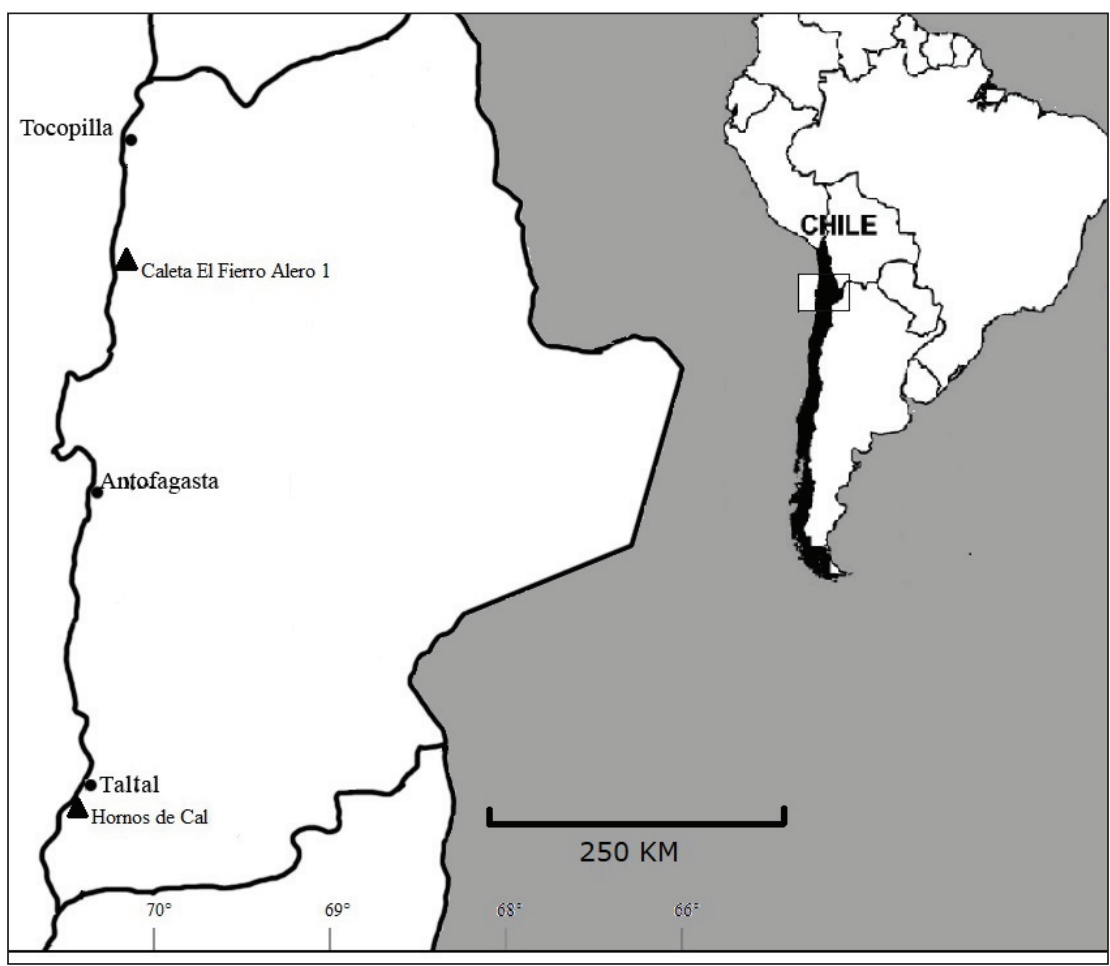

Fig. 2. Ubicación geográfica de los sitios mencionados en el texto 
el programa de calibración OxCal 4.3 (Ramsey, 2017), aplicando la curva MARINE13 (Reimer et al., 2013) y el efecto reservorio para el norte de Chile propuesto por Ortlieb et al. (2011), debido el alto consumo de recursos marinos que poseen los individuos de la zona (Andrade et al., 2015).

\section{RESULTADOS}

\section{Determinación del sexo, estimación de edad y contextualización cronológica}

Para el caso del individuo de Hornos de Cal, se puede decir que se trata de un individuo masculino adulto joven (20-25 años de edad), que presentó un fechado radiocarbónico de $6180 \pm$ 25 años AP (cal 6712 [6375] 6011; UGAMS 30567). En la cronología local, este momento se ha denominado como Arcaico III (Salazar et al., 2015) y corresponde a una etapa marcada por la aparición de densos concheros y restos de fauna terrestre asociados a depósitos habitacionales potentes, generados por grupos especializados en la extracción de los abundantes recursos marinos. Debido a la densidad que presentan los depósitos, se ha planteado que estos grandes concheros habrían funcionado como campamentos base de uso prolongado, desde donde se habrían articulado radios de forrajeo amplios para obtener recursos presentes en zonas alejadas (Castelleti, 2007; Salazar et al., 2015). Cabe mencionar que tanto dentro de la cronología local de Taltal como la regional, no existen otros individuos que hayan sido fechados dentro de este periodo cronológico (Salazar et al., 2015).

Por su parte, el caso del individuo recuperado de CEFA-1 corresponde a un individuo de sexo femenino, adulto joven (30-35 años de edad), con un fechado radiocarbónico de 1360 \pm 25 años AP (cal 978 [697] 460; UGAMS 15622). En la cronología local, este momento corresponde al periodo Intermedio Tardío (Castro, 2014). Como ya se mencionó anteriormente, esta etapa está marcada por la presencia de artefactos de diversos sectores del Norte Grande de Chile, por lo que se ha asumido una intensificación de los contactos con otros grupos humanos, configurándose la costa de Antofagasta como un espacio de contextos multiétnicos. Con respecto a otros individuos reportados en este periodo cronológico, solo se cuenta con la presencia de un individuo al norte de Taltal, en sitio Alero Bandurrias 2 (Andrade et al., 2014), en el sector de Mejillones (Bittmann y Munizaga, 1979), al norte de Tocopilla (Clarot et al., 2014-2015) y de la desembocadura del río Loa (Spahni, 1967), no existiendo referencias sobre la presencia de LSTV en ninguno de los casos. Esto es de esperar ya que estudios recientes realizados por Arriaza et al. (2019), indican una prevalencia de un $0,1 \%$ para enfermedades congénitas en poblaciones arqueológicas que habitaron la costa norte de Chile.

\section{Patologías vertebrales}

Para el individuo recuperado en el sitio Hornos de Cal, se pudo diagnosticar la presencia de una hemi-sacralización unilateral derecha de L5 (Fig. 3a), correspondiente al tipo II-a de Castellvi et al. (1984). Considerando el fechado obtenido, este individuo presentaría el caso más temprano de esta patología para el norte de Chile. Este individuo no presentó ninguna otra condición patológica en las unidades óseas susceptibles de ser analizadas.

En el caso de CEFA-1 (Fig. 3b), se diagnosticó una hemi-sacralización unilateral izquierda de L5, correspondiente al tipo II-a de Castellvi et al. (1984). Además, se presentaron otras patologías correspondientes a crecimiento óseo en la cara anterior y superior de los márgenes de los cuerpos de las vértebras lumbares, espina bífida oculta, adelgazamiento de los cuerpos vertebrales de L4 y L3, provocando desviación, una probable escoliosis leve, y osteofitos en los cuerpos vertebrales lumbares. Todas estas patologías resultan compatibles con la presencia de la sacralización observada. En el caso específico de la espina bífida, no aparecen reportes en la literatura bioarqueológica para la costa de Antofagasta, aunque sí se reporta en grupos de cazadores recolectores de la costa del extremo norte de Chile (Silva-Pinto, Arriaza y Standen, 2010), donde se presentó en 5 de 37 individuos analizados, proponiéndose para este caso, que su origen más probable se encontraría en la ingesta de agua con altos niveles de arsénico, sin que se descarte un posible origen genético de esta enfermedad, ligado a la endogamia, como también se ha mencionado para otras anomalías del desarrollo encontradas en la misma población (Standen et al., 2018). La presencia 


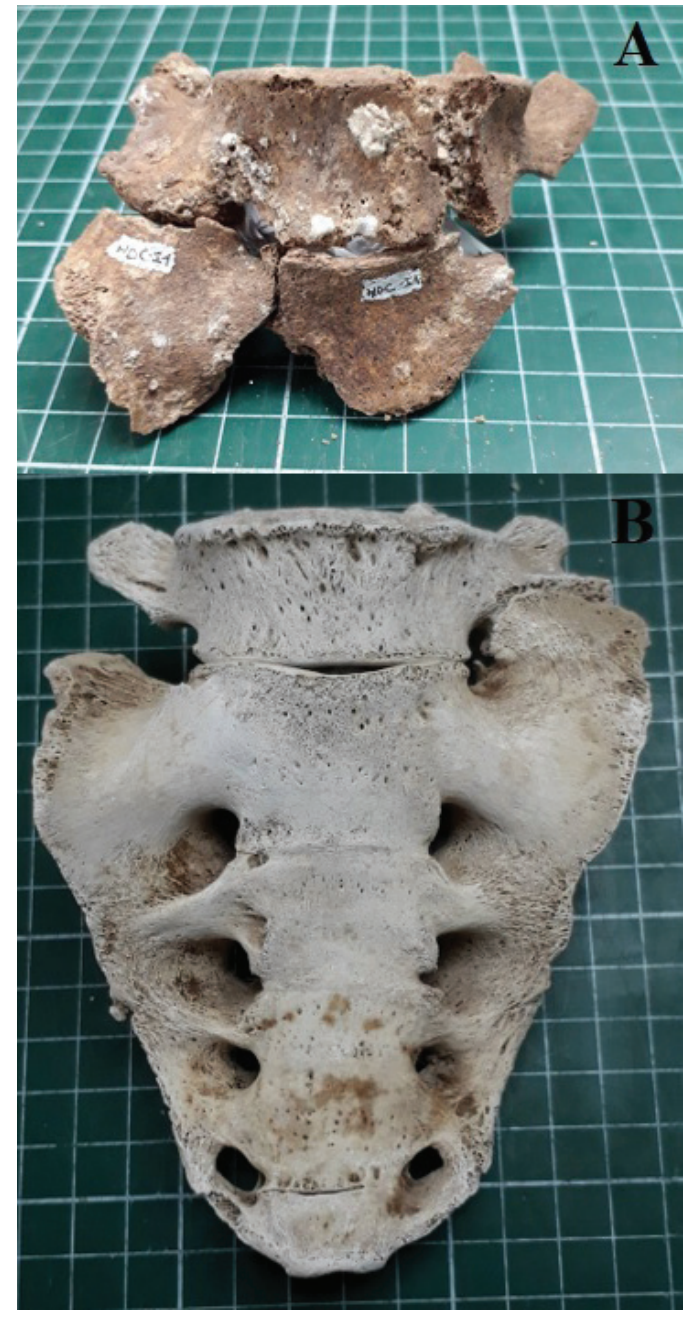

Fig. 3. A. Vista anterior de fragmento de sacro con presencia de sacralización incompleta unilateral derecha de L5 del individuo del sitio Hornos de Cal. 3. B. Vista anterior del sacro con sacralización incompleta de L5 unilateral izquierda del individuo del sitio CEFA-1

de enfermedades vertebrales degenerativas en el caso del individuo recuperado en CEFA-1, son similares con otras observadas en individuos de la costa de Antofagasta (Andrade et al., 2014; Berrios, 2014), por lo que su manifestación puede deberse a que el individuo continuó realizando las labores comunes de su grupo social a pesar de la presencia de sacralización de L5.

\section{DISCUSIÓN}

La presencia de patologías vertebrales es ampliamente conocida en poblaciones humanas prehistóricas del norte de Chile, sin embargo, mayoritariamente corresponden a patologías degenerativas y morfofuncionales, ligadas a una vida dedicada a la extracción de recursos marinos, transporte de peso extracorporal y en algunos casos, de trabajos agrícolas (Andrade et al., 2014; Berrios, 2014; Constanzo, 2013; Henríquez y Arriaza, 2013). Sin embargo, en la bibliografía especializada no se reportan casos que den cuenta de la anomalía aquí presentada, tanto para la zona de estudio, como para otros sectores de la costa norte de Chile (Gerszten, Gerszten y Allison, 2001; Quevedo, 2000; Silva-Pinto, Valenzuela y Sepúlveda, 2013; Standen, Allison y Arriaza, 1984), salvo por la reciente publicación de un caso en Arica, correspondiente a un individuo femenino del periodo Intermedio Tardío (Arriaza et al., 2019).

En primer término, consideramos que esta expresión patológica no puede ser resultado de las condiciones ambientales (altos niveles de arsénico en el agua), ya que el marco geográfico en que habitaron las poblaciones involucradas, así como las actividades que realizaron, fueron relativamente similares a lo largo del tiempo (Andrade et al., 2014). Si fuese así, una mayor cantidad de individuos presentarían LSTV, por lo que la prevalencia en la población seria mayor. Tampoco consideramos que la causa de estas patologías haya sido un evento traumático, ya que no se presentan lesiones en las unidades óseas aledañas, como tampoco se evidencian callos óseos en las zonas lindantes. Específicamente para el caso de la espina bífida oculta observada en CEFA-1 y su asociación a la arsenicosis (Silva-Pinto et al., 2010), no tenemos registros de los niveles de las aguas locales que pudieron ser consumidas por los individuos aquí analizados. Sin embargo, estudios en la población moderna de Antofagasta dan cuenta del contenido de este elemento en aguas y alimentos consumidos actualmente (Cortés, Pino, Atalah, Silva y Jara, 2004; Sancha y Frenz, 2000; Santander, Jamett, Peña, Muñoz y Gras, 1994) y, aunque se reportan varias enfermedades asociadas, ninguna de estas investigaciones menciona la aparición de defectos del desarrollo óseo vertebral (Montalva, 2016; Puga et al., 1973; Rivera y Corey, 1995; Santander et al., 1994). Adicionalmente, un estudio reciente (Tapia et al., 2018) indica que la presencia de arsénico en 
Antofagasta no es natural, sino producto de actividades humanas, como procesos de extracción minera industrializada, desarrollada en la zona durante el siglo XX, por lo que es probable que el contenido de arsénico en las aguas consumidas por la población arqueológica de la costa de Antofagasta haya sido muy bajo e incluso nulo. De esta manera, se descarta la posibilidad de una causa de tipo ambiental.

Considerando todo lo anterior, la explicación más plausible en cuanto a las causas de las expresiones de las LSTV en la población estudiada tendría un origen genético sustentado en las prácticas culturales endogámicas. Nuestra hipótesis se sustenta en el proceso de diferenciación poblacional entre los grupos de Arica y Antofagasta mencionado anteriormente. Creemos que este habría sido fundamental en la configuración genética de los habitantes de la costa de Antofagasta, los cuales no habrían tenido importantes contactos con otros grupos humanos foráneos, por lo menos hasta el periodo Formativo. Sería esperable entonces, observar un aumento en la diversidad del pool génico, lo que se intensificaría aún más en momentos más tardíos de la secuencia cronológica. Sin embargo, se debe tener en consideración el impacto real en términos de transformaciones sociales que dichos contactos efectivamente produjeron en los distintos aspectos culturales de las poblaciones de Antofagasta, los cuales no parecen haber sido significativos (Andrade et al., 2015; Castro, 2004; Pestle 2017; Salazar et al., 2015). A esto, se suman los datos etnográficos e históricos que describen lazos matrimoniales altamente endogámicos en las poblaciones costeras de Antofagasta, las que podrían tener su origen en tradiciones ancestrales, considerando la conformación social y cultural tradicional de los grupos costeros de Antofagasta (Borie et al., 2017; Contreras et al., 2011; Palma, 2012).

Por ende, más allá de encontrarse relativamente aisladas geográficamente, las poblaciones prehistóricas de la costa de Antofagasta habrían además optado por establecer un aislamiento reproductivo con respecto a sus vecinos, el cual habría estado sustentado por un proceso de identidad territorial que tendría sus orígenes en momentos muy tempranos (Salazar et al., 2018), lo cual se habría perpetuado a través de relaciones de parentesco mantenidas de manera tradicional, al menos hasta momentos históricos. El contexto anterior brinda un marco para interpretar las anomalías de desarrollo vertebral como resultado de grupos con prácticas reproductivas altamente endogámicas (Brothwell, 1987; Comsa, Kogalmiceanu y Nalbitoru, 20102011; Goldstein, Aresburg y Nathan, 1976; Groza, Simalcsik, Bejenaru y Simalcsik, 2016; Henneberg y Henneberg, 1999; Titelbaum y Uceda, 2015; Turkel, 1989) entre los que se encontrarían algunas sociedades de cazadoresrecolectores (Macdonald y Hewlett, 1999; Pemberton y Rosenberg, 2014; Walker y Bailey, 2014), tal como sucedería con los habitantes prehispánicos de la costa de Antofagasta.

\section{CONCLUSIONES}

En vista de lo presentado anteriormente, creemos que efectivamente la aparición de las LSTV en las poblaciones de Antofagasta responde a prácticas de entrecruzamiento endogámicas, a pesar de los contactos mantenidos con poblaciones de otras latitudes, especialmente desde periodo Formativo en adelante. Esto se configuraría en una manifiesta elección cultural en términos de la reproducción social y biológica de los grupos humanos. Además, creemos que este reporte abre una nueva ventana hacia la comprensión de los mecanismos sociales de los antiguos habitantes de la costa de Antofagasta. Así, podemos elucubrar que los mecanismos de alianzas matrimoniales observados por los primeros cronistas poseerían una larga data en la zona y que los componentes genéticos externos reportados por Henríquez et al. (2004) corresponderían a un fenómeno relativamente reciente, los que podrían ser efecto de los importantes cambios sociales producto de la instauración de los sistemas coloniales y republicanos (Ballester, San Francisco y Gallardo, 2010; Castelleti et al., 2019; Castelleti, 2019). Esto nos lleva a cuestionarnos el impacto del contacto con poblaciones foráneas en la costa de Antofagasta, lo cual aparentemente no habrían repercutido en los modos de vida ni otras esferas sociales. Lo anterior debe ser continuado en una futura investigación que permita establecer filiaciones de parentesco en base al análisis de ADN antiguo que nos ayude a clarificar nuestras hipótesis. De todas formas, creemos que la presencia 
de esta patología, nos permite no solo ampliar el espectro de las enfermedades que afectaron a los grupos humanos de la costa desértica de Chile en tiempos remotos, sino que también nos ayuda a comprender de mejor manera procesos sociales que implicaban la continuidad biológica del grupo. Finalmente, consideramos que este trabajo contribuye a la búsqueda de nuevos significados en torno a la presencia de patologías ligadas a anomalías del desarrollo esqueletal.

\section{AGRADECIMIENTOS}

Los autores expresan su gratitud a Diego Salazar y Victoria Castro, investigadores responsables de los proyectos FONDECYT 1151203 y 1100951, respectivamente, por permitirnos trabajar con estos restos y publicar los resultados obtenidos. A Leandro Luna, Claudia Aranda y Lía Leyton, por la lectura crítica y aportes a la primera versión del artículo. Finalmente, los autores agradecen a los revisores anónimos por sus importantes comentarios que ayudaron a mejorar la calidad de este trabajo.

\section{LITERATURA CITADA}

Aldunate, C., Castro, V. y Varela, V. (2010). Los Atacamas y el pescado de Cobija. En homenaje al maestro John Víctor Murra. Chungara, 42, 341-347. doi: 10.4067/ S0717-73562010000100039

Andrade, P., Castro, V., y Aldunate, C. (2016). Reconstrucción del modo de vida de individuos del arcaico de la costa arreica del norte de Chile: una aproximación bioarqueológica desde el sitio Copaca 1. Chungara, 48, 73-90. doi:10.4067/S0717-73562016005000007.

Andrade, P., Salazar, D., Urrea, J., y Castro, V. (2014). Modos de vida de los cazadores-recolectores de la costa arreica del norte grande de Chile: una aproximación bioarqueológica a las poblaciones prehistóricas de Taltal. Chungara, 46, 467-491. doi:10.4067/S071773562014000300010

Andrade. P., Fernandes, R., Codjambassis, K., Urrea, J., Olguín, L., Rebolledo, S., Lira, F., Aravena, C., y Berrios, M. (2015). Subsistence continuity linked to consumption of marine protein in the formative period in the interfluvic coast of northern Chile: re-assessing contacts with agropastoral groups from highlands. $R a$ diocarbon, 57, 679-688. doi:10.2458/azu rc.57.18448

Ardiles, H., Ballester, B., y Clarot, A. (2011). Elección de dieta en poblaciones pasadas costeras de la II Región: una mirada multidisciplinaria. Informes FAIP, 14, 83110.

Arias, M. y Herrera, M. J. (2012). Caracterización del modo de vida, salud y dieta de las poblaciones de los cementerios Caserones-Tarapacá 40 y Cáñamo 3 (Período Formativo, Región de Tarapacá). En Actas del XVIII Congreso Nacional de Arqueología Chilena (pp. 45-54) Arica, Chile.
Arriaza, B., Standen, V., Soto, L., y Aravena, N. (2019). A prehistoric case of developmental defects of the spine in a young adult female from the Atacama Desert. International Journal of Paleopathology, 29: 346-349. doi: 10.1002/oa. 2738

Aufderheide, A., y Rodríguez-Martín, C. (1998). The Cambridge encyclopedia of human paleopathology. Cambridge, Reino Unido: Cambridge University Press.

Ballester, B. (2017). Parentesco y política de los cazadores, pescadores y recolectores marinos del Desierto de Atacama según crónicas, documentos históricos y restos materiales. F. Gallardo, B. Ballester y N. Fuenzalida. (Eds.). Monumentos funerarios de la costa del Desierto de Atacama. Los cazadores. Recolectores marinos y sus intercambios (500 a.C.-700 d.C.) (pp: 47-50). Santiago, Chile: Andros.

Ballester, B. (2018). El Médano rock art style: Izcuña paintings and the marine hunter-gatherers of the Atacama Desert. Antiquity, 92, 132-148. doi:10.15184/ aqy.2017.185

Ballester, B., Clarot, A., y Llagostera, A. (2014). El cementerio de Auto Club de Antofagasta y la sociedad litoral entre los 1000 y 1450 d.c. Hombre y Desierto, 18, 187212

Ballester, B., San Francisco, A., y Gallardo, F. (2010). Modo de vida y economía doméstica de las comunidades cazadoras recolectoras costeras del desierto de Atacama en tiempos coloniales y republicanos. Taltalia, 3, 21-32.

Ballester, B., Vidal, E., Calás, E., Gallardo, F., Aguilera, P., Pellegrino, C., y Clarot, A. (2018). Un enclave arcaico tardío en la aguada costera de Guala Guala (desierto de Atacama, Norte de Chile). Chungara, 50, 349-367. doi:10.4067/S0717-73562018005000702

Ballester, B., y Clarot, A. (2014). La gente de los túmulos de tierra. Estudio, conservación y difusión de colecciones arqueológicas de la comuna de Mejillones. Santiago, Chile: Gráfica Marmor

Ballester, B., y Gallardo, F. (2017). La versatilidad del parentesco en la reproducción social: El caso de los cazadores-pescadores marinos del desierto de Atacama (siglos XVI-XIX, norte de Chile). Boletín de la Sociedad Chilena de Arqueología, 47, 7-28.

Barnes, E. (1994). Developmental defects of the axial skeleton in paleopathology. Colorado, Estados Unidos: University Press of Colorado.

Barnes, E. (2008). Congenital Anomalies. En R. Pinashiy S. Mays (Ed). Advances in human paleopathology (pp. 329-362). Chichester, Reino Unido: John Wiley y Sons. doi:10.1002/9780470724187.ch15

Berenguer, J. (2009). Las pinturas del Médano, norte de Chile: 25 años después Motsny y Niemeyer. Boletin del Museo Chileno de Arte Precolombino, 14, 57-95. doi:10.4067/S0718-68942009000200004

Berríos, M. (2014). Paleopatologías degenerativas de la columna vertebral de restos bioantropológicos de cazadores-recolectores provenientes de la costa arreica. Comparación con una colección similar de la costa del Norte Semiárido (Memoria de pregrado). Universidad de Concepción, Concepción, Chile.

Bittmann, B., y Munizaga, J. (1979). El arco en América: evidencia temprana y directa de la cultura Chinchorro (Norte de Chile). Indiana, 5, 229-250.

Blanco, J., Correa, I., Flores, C., y Pimentel, G. (2017). La extracción prehispánica de recursos minerales en el internodo Quillagua-Costa, desierto de Atacama. Estudios Atacameños, 56, 77-102. doi:10.4067/S071810432017005000003

Borie, C., Power, X., Parra, S., Salinas, H., Rostan, P., 
Galarce, P., ... y Traverso, F. (2017). Tras la huella del sílice pampino. Nuevas metodologías para el rastreo de las áreas fuente de aprovisionamiento lítico en Taltal. Estudios Atacameños, 56, 103-131. doi:10.4067/ S0718-10432017005000005

Bron. J., van Royen, B., y Wuisman, P. (2007). The clinical significance of lumbosacral transitional anomalies. Acta Orthopaedica Belgica, 73, 687-695.

Brothwell, D. (1987). Desenterrando huesos. La excavación, tratamiento y estudio de los restos del esqueleto humano. Ciudad de México, México: Fondo de Cultura Económica.

Buikstra, J., y Ubelaker, D. (1994). Standards for data collection from human skeletal remains. Arkansas Archeological Survey Research Series 44. Arkansas, Estados Unidos: Fayetteville.

Caldwallader, L., y Arce, S. (2012). Proyecto de investigación arqueológica Samaca. Informe de los trabajos del campo realizados durante la temporada 2010. Lima, Perú: Ministerio de Justicia

Campo, M. (2003). Paleopatología de a columna vertebral. En A. Isidro y A. Malgosa (Ed.). Paleopatología. la enfermedad no escrita (pp. 163-194). Barcelona, España: Masson.

Carapuco, M., Nóvoa, A., Bobola, N., y Mallo, M. (2005). Hox genes specify vertebral types in the presomitic mesoderm. Genes \& Development, 19, 2116-2121. doi:10.1101/gad.338705

Carrasco, C., Carmona, J., Ballester, B., y Niemeyer, H. (2013). De pipas y sustancias: costumbres fumatorias durante el periodo Formativo en el litoral del desierto de Atacama (Norte de Chile). Latin American Antiquity, 26, 143-161. doi: 10.7183/1045-6635.26.2.143

Castelleti, J. (2007). Patrón de asentamiento y uso de recursos a través de la secuencia ocupacional prehispana en la costa de Taltal. (Tesis de Magíster). Universidad Católica del Norte-Universidad de Tarapacá. San Pedro de Atacama, Chile.

Castelleti, J. (2019). History of the landscape and rural territory among the indigenous families of Paposo and Taltal on the Atacama Desert coast. Journal of Family History. doi:10.1177/0363199019874010. En prensa.

Castelleti, J., Villarroel, M., Almendares, H., y Mercado, P. (2019). Historia del paisaje rural entre las familias de Paposo y Taltal. Diálogo Andino 58: 77-88. doi:10.4067/S0719-26812019000100077

Castelleti, J., y Maltrain, G. (2010). El Formativo de Taltal y el patrón de asentamiento local. En Actas del XVII Congreso Nacional de Arqueología Chilena (pp. 165-176). Valdivia, Chile: Ediciones Kultrún.

Castellvi, A., Goldstein, L., y Chan, D. (1984). Lumbosacral transitional vertebrae and their relationship with lumbar extradural defects. Spine, 9(5), 493-495. doi:10.1097/00007632-198407000-00014

Castro, V. (2014). Pre-Hispanic cultures in the Atacama desert: a Pacific coast overview. En N. Sanz, B. Arriaza y V. Standen. The Chinchorro culture. A comparative perspective. The archaeology of the earliest human mummification (pp. 11-34). Arica, Chile: Andros.

Castro, V., Aldunate C., y Varela, V. (2012). Paisajes culturales de Cobija, costa de Antofagasta, Chile. Revista Chilena de Antropología, 26, 97-128. doi: 10.5354/07191472.2013.26556

Clarot, A., Méndez, A., y Ballester, B. (2014-2015). Un costeño del desierto de Atacama: acercamiento al modo de vida en el periodo Intermedio Tardío mediante imagenología e isótopos estables. Taltalia, 7-8, 39-60
Cocilovo, J., Varela, H., Costa-Junqueira, M., y Quevedo, S. (2005). Los pescadores arcaicos de la desembocadura del río Loa (norte de Chile): el sitio Caleta Huelén 42. Chungara, 37, 5-19. doi: 10.4067/S071773562005000100002

Cocilovo, J., Varela, H., Quevedo, S., Standen, V., y CostaJunqueira, M. (2004). La diferenciación geográfica de la población humana arcaica de la costa norte de Chile (5000-3000 AP) a partir del análisis estadístico de rasgos métricos y no métricos del cráneo. Revista Chilena de Historia Natural, 77, 679-693. doi:10.4067/S0716078X2004000400010

Comsa, A., Kogalniceanu, R., y Nalbitoru, A. (2010-2011). The burial beneath the getic earthen defence wall of the dava from Radovanu - Gorgana Intâi. Thraco-dacíca, $X X V-X X V I, 31-38$

Constanzo, D. (2013). Una aproximación a la reconstrucción de los modos de vida a partir de las enfermedades degenerativas articulares en la columna vertebral en las poblaciones de la costa arreica en el norte grande de Chile (memoria de pregrado). Universidad de Concepción, Concepción, Chile

Contreras, R., Núñez, P., Llagostera, A., Cruz, J., San Francisco, A., Ballester, B., ... y Becerra, G. (2011). Un conglomerado del periodo Arcaico costero Medio del área Taltal Paposo, Norte de Chile. Taltalia 4, 7-31.

Correa, I., Carrasco, C., Ballester, B., y Gallardo, F. (2018). Efectos colaterales de la transición al Formativo: una nueva culinaria entre los cazadores-recolectores marinos del desierto de Atacama. Chungara, 50, 87-106. doi:10.4067/S0717-73562018005000101

Cortés, S., Pino, P., Atalah, E., Silva, C., y Jara, M. (2004). Exposición a arsénico ambiental en niños de Antofagasta, II región, Chile. Arsénico y sus consecuencias en la Salud y Ambiente, 85, 1-14.

Costa-Junqueira, M. (2001). Modalidades de enterramientos humanos arcaicos en el norte de Chile. Chungara, 33, 55-62. doi:0.4067/S0717-73562001000100009

Costa-Junquiera, M., y Sanhueza, J. (1976). Poblaciones precolombinas de la costa norte de Chile: Restos óseos humanos de los cementerios Punta Blanca y Auto Club (Antofagasta). Seminario Medio Integral. Departamento de Ciencias Sociales. Universidad del Norte Antofagasta. Antofagasta, Chile

Chang, H., y Nakagawa, H. (2004). Altered function of lumbar nerve roots in patients with transitional lumbosacral vertebrae. Spine, 29(15), 1632-1635. doi:0.1097/01. BRS.0000132319.43140.D3

de Bruin, F., Ter Hortst, S., Bloem, J., van den Berg, R., de Hooge, M., van Gaalen, F., ... y Reijnierse, M. (2017). Prevalence and clinical significance of lumbosacral transitional vertebra (LSTV) in young back pain population with suspected axial spondyloarthritis: results of spondyloarthritis caught early cohort. Skeletal Radiology, 46, 633-639. doi:10.1007/ s00256-017-2581-1

Flores, C., Figueroa, V., y Salazar, D. (2016). Middle Holocene production of mussel Shell fishing artifacts on the coast of Taltal ( $25^{\circ}$ Lat South), Atacama Desert, Chile. The Journal of Island and Coastal Archaeology, 11: 411-424. doi: 10.1080/15564894.2015.1105884

FONDECYT 1151203. (2017). Guía de Campo. Seminario Trayectoria Histórica de los Cazadores Recolectores de la Zona de Taltal y Paposo.

Gallardo, F. (2017). Arqueología de los intercambios recíprocos: costa y oasis del río Loa Medio e Inferior, épica formativa (500 cal. A.C.-700 d.C.). En F. Gallardo, B. Ballester y N. Fuenzalida (Eds.). Monumentos Funera- 
rios de la Costa del Desierto de Atacama. Los Cazadores. Recolectores Marinos y sus Intercambios (500 a.C.700 d.C.) (pp: 15-24). Santiago, Chile: Andros.

Gallardo, F., Ballester, B., y Fuenzalida, N. (2017). Monumentos funerarios y flujos de información social costera. En F. Gallardo, B. Ballester y N. Fuenzalida (Eds.). Monumentos Funerarios de la Costa del Desierto de Atacama. Los Cazadores-Recolectores Marinos y sus Intercambios (500 a.C. - 700 d.C.) (pp. 39-46). Santiago, Chile: Andros

Gerszten, P., Gerszten, E., y Allison, M. (2001). Diseases of the Spine in South American Mummies. Neurosurgery, 48(1), 208-213. doi:10.1097/00006123-20010100000039

Goldstein, M., Arensburg, B., y Nathan, H. (1976). Pathology of bedouin skeletal remains from two sites in Israel. American Journal of Physical Anthropology, 45, 621640. doi: 10.1002/ajpa.1330450329

Goodman, R., y Gorlin, R. (1983). The Malformed Infant and Child: An Illustrated Guide. New York, Estados Unidos: Oxford University Press.

Groza, V., Simalcsik, A., Bejenaru, L., y Simalcsik, R. (2016). Spina bifida occulta in medieval and postmedieval times in Eastern Romania. Memoirs of the Scientific Sections of the Romanian Academy, XXXIX, 103-115

Henneberg, R., y Henneberg, M. (1999). Variation in the closure of the sacral canal in the skeletal sample from Pompeii, Italy, 79 AD. Perspectives in Human Biology, 4, 177-188

Henríquez, M., Moraga, M., Llop, E., y Rothhammer, F. (2004). Caracterización genético molecular de habitantes de Caleta Paposo, último reducto Chango en Chile. Revista Médica de Chile 132, 663-672. doi:10.4067/ S0034-98872004000600002

Henríquez, M., y Arriaza, B. (2013). Distribución y frecuencia de nódulos de Schmörl en la columna vertebral de poblaciones prehispánicas de Arica: ¿Indicadores de carga laboral? Chungara 45, 311-319. doi:10.4067/ S0717-73562013000200007

Hsieh, C., Vanderford, J., Moreau, S., y Prong, T. (1999). Lumbosacral transitional segments: Classification, prevalence, and effect on disk height. Journal of Manipulattive and Physiological therapeutics, 23(7), 483-489. doi:10.1067/mmt.2000.108817

Ibacache, M. (2014). Aproximación al estudio de los rasgos craneométricos de la costa arreica del norte de Chile (Memoria de pregrado). Universidad de Concepción, Concepción, Chile

Kong, C., Park, J., y Park, J. (2008). Sacralization of L5 in radiological studies of degenerative spondylolisthesis at L4-L5. Asian Spine, 2(1), 34-37. doi: 10.4184/ asj.2008.2.1.34

Kozameh, L., Testa, N., y Tessone, A. (2011). Osteobiografía de dos cazadores de ambientes marinos de la Isla Grande de Tierra del Fuego. Revista de la Escuela de Antropología, XVII, 201-216.

Kumari, S., Kumari, R., Panchal, P., Prasad, A., y Britto, N. (2016). Study of sacralization of fifth lumber vertebra. Journal of Dental and Medical Sciences, 15(11), 58-61. doi:10.9790/0853-1509011821

Leboeuf, C., Kimber, D., y White, K. (1989). Prevalence of spondylolisthesis, transitional anomalies and low intercrestal line in a chiropractic patient population. Journal of Manipulative and Physiological Therapeutics, 12, 200-204.

LeClair, E., Bonfiglio, L., y Tuan, R. (1999). Expression of the paired-box genes Pax-1 and Pax-9 in limb skeleton development. Developmental Dynamics, 214, 101-115. doi: 0.1002/(SICI)1097-0177(199902)214:23.0.CO;2-4 Letelier, J., y Castro, V. (2017). Changos en el Puerto de Cobija. Transformaciones sociales durante el siglo XIX. Revista Española de Antropología Americana, 47, 127 142. doi:10.55209/REAA.61974

Llagostera, A. (2005). Culturas costeras precolombinas en el norte chileno: secuencia y subsistencia de las poblaciones arcaicas. En E. Figueroa (Ed.). Biodiversidad marina: Valoración, uso y perspectivas (pp: 107-148). Santiago, Chile: Editorial Universitaria.

Llagostera, A., y Cruz, J. (2010). Evaluación del recurso arqueológico de la costa del desierto de Atacama para diversificar los destinos del turismo de intereses especiales de la región de Antofagasta. Taltalia, 3, 45-55

MacDonald, D., y Hewlett, B. (1999). Reproductive interest and forager mobility. Current Anthropology, 40, 501523. doi:10.1086/200047

Mallo, M., Wellik, D., y Deschamps, J. (2010). Hox genes and regional patterning of the vertebrate body plan. Developmental Biology, 344(1), 7-15. doi: 10.1016/j. ydbio.2010.04.024

Miranda, P. (2011). Estudio paleopatológico de un entierro humano en el salar de Patos Grandes, Puna de Salta, Argentina. Cazadores Recolectores del Cono Sur, 5, 163-177.

Montalva, M. (2016). Enfermedad de Bowen tras 45 años de exposición a arsénico en Antofagasta: comparación con una población no expuesta de la Región Metropolitana (Tesis de magister). Universidad de Chile, Santiago, Chile.

Moreno, S., del Rio, P., Baltanás, P., y Cía, P. (2016). Síndrome de Bertolotti: a propósito de un caso. Revista Colombiana de Reumatología, 23(3), 200-203. doi:10.1016/j.rcreu.2015.12.007

Munizaga, J. (1980). Restos óseos humanos de la costa norte de Chile: Análisis de cementerios disturbados de Cobija, II Región, Chile. En B. Bittmann, M.T. Ahumada y C. Moragas (Eds.). Proyecto de investigación interdisciplinaria en la costa centro-sur andina, Vol I. (pp: 199-216) Universidad del Norte. Antofagasta, Chile: Imprenta Universitaria.

Murlimanju, B., Prabhu, L., Pai, M., Ganeshkumar, C., y Sarvepalli, A. (2011). Lumbosacral transitional vertebrae: a case report and clinical implications. International Journal of Morphology, 29, 1123-1125. doi:10.4067/S0717-95022011000400008

Nardo, L., Alizai, H., Virayavanich, W., Lui, F., Hernandez, A., Lynch, J., Nevitt, C., Lane, N., y Link, T. (2012). Lumbosacral transitional vertebrae: association with low back pain. Radiology, 265, 497-503. doi:10.1590/ S0004-282X2009000200018

Núñez, L., Zlatar, V., y Núñez, P. (1975). Caleta Huelén 42: una aldea temprana en el norte Chile. (Nota preliminar). Hombre y Cultura, 2, 67-103.

Olguín, L., Salazar, D., y Jackson, D. (2014). Tempranas evidencias de navegación y caza de especies oceánicas en la costa Pacífica de Sudamérica (Taltal 7000 años cal ap). Chungara 46, 177-192. doi:10.4067/S071773562014000200002

Ortlieb, L., Vargas. G., y Saliege, J.F. (2011). Marine radiocarbon reservoir effect along Northern ChileSouthern Peru coast $\left(14-24^{\circ} \mathrm{S}\right)$ throughout the Holocene. Quaternary Research, 75, 91-203. doi:10.106/j. yqres. 201007018

Otani, K., Konno, S., y Kikuchi, S. (2001). Lumbosacral transitional vertebra and nerve root symptoms. The Journal of Bone and Joint Surgery. British Volume 83, 1137-1140. doi: 10.1302/0301-620X.83B8.11736 
Palma, C. (2012). Una primera aproximación al estudio de patrones de asentamiento durante el Periodo Intermedio Tardio en la costa de Tocopilla (memoria de pregrado). Universidad de Chile, Santiago, Chile

Paraskeas, G., Tzika, M., y Kitsoulis, P. (2013). Lumbosacral transitional vertebra associatted with sacral spina bífida occulta: a case report. Acta Médica, 56, 126-129. doi:10.14712/18059694.2014.21

Pemberton, T., y Rosenberg, N. (2014). Population-genetic influences on genomic estimates of the inbreeding coefficient: A global perspective. Human Heridity, 77, 37 48. doi:10.1159/000362878

Pestle, W. (2017). Living, eating and dying in formative period Atacama Desert. En F. Gallardo, B. Ballester y N. Fuenzalida (Eds.). Monumentos funerarios de la costa del Desierto de Atacama. Los cazadores-recolectores marinos y sus intercambios (500 a.C.-700 d.C.) (pp. 209-224). Santiago, Chile: Andros Editores.

Pestle, W., Torres-Rouff, C., Ballester, B., Gallardo, F., y Clarot, A. (2015). Mobility and exchange among marine hunter-gatherer and agropastoralist communities in the Formative period Atacama Desert. Current Anthropology, 56, 121-133. doi:10.1086/679594

Pimentel, G., Rees, C., de Souza, P., y Ayala, P. (2010). Estrategias de movilidad del período Formativo en la depresión intermedia, desierto de Atacama. En Actas del XVII Congreso Nacional de Arqueología Chilena (pp. 1353-1364). Valdivia, Chile: Ediciones Kultrún.

Pliscoff, P., y Luebert, F. (2008). Los ecosistemas terrestres. En J. Rodovira, J. Ugalde y M. Stutzin (Eds.). Biodiversidad de Chile: Patrimonio y Desafios (pp. 74-87). Santiago, Chile: Ocho Libros.

Puga, F., Olivos, P., Greiber, R., González, I., Heras, E., Barrera, S., y González, E. (1973). Hidroarsenicismo crónico (intoxicación arsenical crónica) en Antofagasta. Estudio epidemiológico y clínico. Revista Chilena de Pediatría, 44, 215-223. doi:10.4067/S037041061973000300002

Quevedo, S. (2000). Patrones de actividad a través de las patologías en población arcaica de Punta Teatinos, Norte Semiárido de Chile. Chungara 32(1), 11-21. doi:10.4067/S0717-73562000000100004

Quezada. J., Cerda, J., y Cerda, A. (2010). Efectos de la tectónica y el clima en la configuración morfológica del relieve costero del norte de Chile. Andean Geology, 37, 78-109. doi:10.4067/S0718-71062010000100004

Quinonez, S., y Innis, J. (2014). Human HOX gene disorders. Molecular Genetics and Metabolism, 111, 4-15. doi:10.1016/j.ymgme.2013.10.012

Ramsey, C. B. (2017). OxCal Project, Version 4.3.

Reimer, P. J., Bard, E., Bayliss, A., Beck, J. W., Blackwell, P. G., Ramsey, C. B., ... y Grootes, P. M. (2013). IntCal13 and Marine13 radiocarbon age calibration curves 0-50,000 years cal BP. Radiocarbon, 55(4), 1869-1887. doi:10.2458/azu_js_rc.55.16947

Rivara, M., y Corey, G. (1995). Tendencia del riesgo de morir por cánceres asociados a la exposición crónica del arsénico, II Región de Antofagasta 1950-1993. Cuadernos de Medicina Social, 36(4), 39-51.

Roberts, C., y Manchester, K. (2005). The Archaeology of Disease. Sparkford, Reino Unido: Sutton Publishing.

Rojas, M., y Walker, L. (2012). Malformaciones congénitas: Aspectos generales y genéticos. International Journal of Morphology, 30, 1256-1265. doi: 10.4067/S071795022012000400003

Salazar, D., Arenas, C., Andrade, P., Olguín, L., Torres, J., Flores, C., Vargas, G., Rebolledo, S., Borie, C., Sandoval, C., Silva, C., Delgado, A., Lira, N., y Robles,
C. (2018). From the use of space to territorialisation during the Early Holocene in Taltal, coastal Atacama Desert, Chile. Quaternary International, 473, 225-241. doi:10.1016/j.quaint.2017.09.035

Salazar, D., Figueroa, V., Andrade, P., Salinas, H., Olguín, L., Power, X., Rebolledo, S., Parra, S., Orellana, H., y Urrea, J. (2015). Cronología y organización económica de las poblaciones arcaicas de la costa de Taltal. Estudios Atacameños, 50, 7-46. doi:10.4067/S071810432015000100002

Salazar, D., Jackson, D., Guendón, J., Salinas, H., Morata, D., Figueroa, V., Manríquez, G., y Castro, V. (2011). Early evidence (ca. 12.000 B.P.) for iron oxide mining on the Pacific coast of South America. Current Anthropology, 52(3), 463-475. doi:10.1086/659426

Salazar, D., Palma, C., Salinas, H., Fuentes, F., Guendón, J., y Castellón, C. (2010). Reconstrucción de la secuencia ocupacional de la quebrada de Mamilla, costa de Tocopilla, norte de Chile. Werkén, 13, 323-346.

Salgan, L., Tucker, H., Luna, L., Aranda, C., y Gil, A. (2012). Estudios arqueológicos y bioarqueológicos en la cuenca media del río Malargüe (provincia de Mendoza): el sitio Bajada de las Tropas 1. Relaciones de la Sociedad Argentina de Antropología, XXXVII, 113-135.

Sancha, A., y Frenz, P. (2000). Estimate of the current exposure if the urban population of northern Chile to arsenic. En Proceeding of the Santiago Symposium. IAHS Publication 260, 3-8.

Santander, M., Jamett, A., Peña, L., Muñoz, L., y Gras, N. (1994). Arsénico en el cabello de habitantes del norte de Chile. Interciencia, 5, 258-263.

Sekharappa, V., Amritanand, R., Krishnan, V., y Samuel, K. (2014). Lumbosacral transition vertebra: prevalence and its significance. Asian Spine, 8, 51-58. doi:10.4184/ asj.2014.8.1.51

Silva-Pinto, V., Arriaza, B., y Standen, V. (2010). Evaluación de la frecuencia de espina bífida oculta y su posible relación con el arsénico ambiental en una muestra prehispánica de la Quebrada de Camarones, norte de Chile. Revista Médica de Chile, 138, 461-469. doi:10.4067/ S0034-98872010000400010

Silva-Pinto, V., Valenzuela, D., y Sepúlveda, M. (2013). Paleopatología osteoarticular en Chinchorro. Revisión de un caso y discusión sobre el autocuidado en la prehistoria de Arica, norte de Chile. Revista Médica de Chile, 141, 637-643. doi:10.4067/S003498872013000500012

Spahni, J. (1967). Recherches acheologiques a l'embochure du rio Loa (cote du Pacifique Chili). Journal de la Societe de Americanistes, 56, 179-239. doi:10.3406/ jsa.1967.2276

Standen, V., Allison, M., y Arriaza, B. (1984). Patologías óseas de la población Morro-1, asociadas al complejo Chinchorro: Norte de Chile. Chungara, 13, 175-185

Standen, V., Santoro, C., Arriaza, B., Valenzuela, D., Coleman, D., y Monsalve, S. (2018). Prehistoric polydactylism: Biological evidence and rock art representation from the Atacama Desert in northern Chile. International Journal of Paleopathology, 22, 54-65. doi:10.1016/j.ijpp.2018.05.005

Tang, M., Yang, X., Yang, S., Han, P., Ma, Y., Yu, H., y Zhu, B. (2014). Lumbosacral transitional vertebra in a population-based study of 5860 individuals: Prevalence and relationship to low back pain. European Journal of Radiology, 83, 1679-1682. doi:10.1016/j. ejrad.2014.05.036

Tapia, J., Valdés, J., Orrego, R., Tchernitchin, A., Dorador, C., Bolados, A., y Harrod, C. (2018). Geologic 
and anthropogenic sources of contamination in settled dust of a historic mining port city in northern Chile: health risk implications. PeerJ 6, e4699. doi:10.7717/ peerj.4699

Taskaynatan, M., Izci, Y., Ozgul, A., Hazneci, B., Dursun, H., y Kalyon, T. (2005). Clinical significance of congenital lumbosacral malformations in young male population with prolonged low back pain. Spine, 30, 210-213. doi:10.1097/01.brs.0000158950.84470.2a

Titelbaum, A., y Uceda, S. (2015). A rare case of os odontoideum from an Early Intermediate period tomb at the Huacas de Moche, Peru. International Journal of Paleopathology, 11, 23-29. doi:101016/jijpp201508001

Torres-Rouff, C. (2017). Fragmentos del cuerpo: Una perspectiva bioarqueológica sobre la vida de personas en la región de Antofagasta (cementerios de túmulos). En F. Gallardo, B. Ballester y N. Fuenzalida (Eds.). Мопиmentos Funerarios de la Costa del Desierto de Atacama. Los Cazadores-Recolectores Marinos y sus Intercambios (500 a.C. - 700 d.C.) (pp. 199-208). Santiago, Chile: Andros Impresores.

Turkel, S. (1989). Congenital abnormalities in skeletal populations. En M. Iscan y K. Kennedy (Eds.) Recons- truction of Life from the Skeleton (pp. 109-128). Nueva York, Estados Unidos: Alan Liss.

Varela, V. (2008). La cerámica arqueológica de Taltal. Taltalia, 2, 119-128.

Vargas, G., Palacios, C., Reich, M., Luo, S., Shen, C., González, G., y Wu, Y. (2011). U-series dating of coseismic gypsum and submarine paleoseismology of active faults in Northern Chile $\left(23^{\circ} \mathrm{S}\right)$. Technophysics, 497, 34-44. doi: 10.1016/j.tecto.2010.10.017

Vargas, G., Rutland, J., y Ortlieb, L. (2006). ENSO tropicalextratropical climate teleconnections and mechanism for Holocene debris flows along the hyperaid coast of western South America $\left(17^{\circ}-24^{\circ} \mathrm{S}\right)$. Earth and Planetary Science Letters, 249, 467-483. doi:10.1016/j.eps1.2006.07.022

Vergauwen, S., Parizel, P., van Breusegem, L., van Goethem, J., Nackaerts, Y., van den Hauwe, L., y de Schepper, A. (1997). Distribution and incidence of degenerative spine changes in patients with a lumbo-sacral transitional vertebra. European Spine Journal, 6, 168-172. doi:10.1007/BF01301431

Walker, R., y Bailey, D. (2014). Marrying kin in small-scale societies. American Journal of Human Biology, 26, 384-388. doi:10.1002/ajhb.22527 